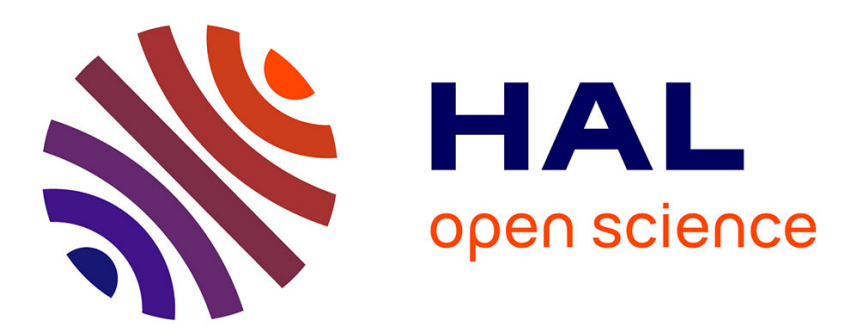

\title{
Vertebral rotation estimation from frontal X-rays using a quasi-automated pedicle detection method
}

Shahin Ebrahimi, Laurent Gajny, Claudio Vergari, Elsa D Angelini, Wafa Skalli

\section{- To cite this version:}

Shahin Ebrahimi, Laurent Gajny, Claudio Vergari, Elsa D Angelini, Wafa Skalli. Vertebral rotation estimation from frontal X-rays using a quasi-automated pedicle detection method. European Spine Journal, 2019, 28, pp.3026-3034. 10.1007/s00586-019-06158-z . hal-02523579

\section{HAL Id: hal-02523579 \\ https://hal.science/hal-02523579}

Submitted on 29 Mar 2020

HAL is a multi-disciplinary open access archive for the deposit and dissemination of scientific research documents, whether they are published or not. The documents may come from teaching and research institutions in France or abroad, or from public or private research centers.
L'archive ouverte pluridisciplinaire HAL, est destinée au dépôt et à la diffusion de documents scientifiques de niveau recherche, publiés ou non, émanant des établissements d'enseignement et de recherche français ou étrangers, des laboratoires publics ou privés. 


\title{
Vertebral rotation estimation from frontal $\mathrm{X}$-rays using a quasi-automated pedicle detection method
}

\author{
Shahin Ebrahimi ${ }^{1} \cdot$ Laurent Gajny $^{1} \quad$ Claudio Vergari $^{1} \cdot$ Elsa D. Angelini ${ }^{2,3} \cdot$ Wafa Skalli $^{1}$
}

\begin{abstract}
Purpose Measurement of vertebral axial rotation (VAR) is relevant for the assessment of scoliosis. Stokes method allows estimating VAR in frontal X-rays from the relative position of the pedicles and the vertebral body. This method requires identifying these landmarks for each vertebral level, which is time-consuming. In this work, a quasi-automated method for pedicle detection and VAR estimation was proposed.

Method A total of 149 healthy and adolescent idiopathic scoliotic (AIS) subjects were included in this retrospective study. Their frontal X-rays were collected from multiple sites and manually annotated to identify the spinal midline and pedicle positions. Then, an automated pedicle detector was developed based on image analysis, machine learning and fast manual identification of a few landmarks. VARs were calculated using the Stokes method in a validation dataset of 11 healthy (age 6-33 years) and 46 AIS subjects (age 6-16 years, Cobb $10^{\circ}-46^{\circ}$ ), both from detected pedicles and those manually anno-tated to compare them. Sensitivity of pedicle location to the manual inputs was quantified on 20 scoliotic subjects, using 10 perturbed versions of the manual inputs.

Results Pedicles centers were localized with a precision of $84 \%$ and mean difference of $1.2 \pm 1.2 \mathrm{~mm}$, when comparin with manual identification. Comparison of VAR values between automated and manual pedicle localization yielded a signed dif-ference of $-0.2 \pm 3.4^{\circ}$. The uncertainty on pedicle location was smaller than $2 \mathrm{~mm}$ along each image axis. Conclusion The proposed method allowed calculating VAR values in frontal radiographs with minimal user intervention and robust quasi-automated pedicle localization.
\end{abstract}

Keywords Vertebral axial rotation $\cdot$ Scoliosis $\cdot$ X-rays $\cdot$ Machine learning $\cdot$ Pedicle detection

Laurent Gajny

Laurent.gajny@ensam.eu

Extended author information available on the last page of the article

\section{Introduction}

Adolescent idiopathic scoliosis (AIS) is a three-dimensional deformity of the spine. While Cobb angle measurements on frontal radiographs are widely used to assess scoliosis 
severity, such 2D measurements are affected by projection bias, and do not take into account the patient's transverse plane $[1,2]$. 3D reconstruction from low dose biplanar $\mathrm{X}$-rays is now routinely used in clinics. 3D reconstruction methods such as [3] have been widely validated, and their clinical relevance is more and more underlined, for diagnosis and early assessment of progressive scoliosis [4-9], and for the evaluation of orthopedic treatments $[10,11]$. In particular, vertebral axial rotations (VARs) can be accurately assessed. In [12], the authors reported a reproducibility of $6.1^{\circ}$ on severe scoliotic subjects. However, low-dose biplanar X-ray systems are not yet generalized. Therefore, assessing the three-dimensional aspect of AIS from a single radiograph is of great interest.

The most natural alternative is the study of a single frontal radiograph. Indeed, by observing the relative position and radiographic appearance of vertebral bodies, pedicles and spinous process in frontal X-rays, vertebral rotations (axial and frontal) can also be estimated. Stokes et al. [13] proposed a quantitative method to approximate VAR from the position of pedicles and vertebral body on frontal radiographs. They reported a 95\% confidence interval for this estimate of $7.1^{\circ}$. However, the method required a tedious manual annotation of both pedicle and vertebral body centers. Thus, our study aims at providing a quasi-automatic tool for VAR quantitative assessment based on pedicle detection in single frontal radiographs of scoliotic patients. This study could secondly be used to automatically improve current 3D spine models.

Pedicles have specific visual characteristics on frontal radiographs, and therefore have the potential of being automatically detected using image processing. Previous works [14-17] proposed various deformable models for pedicle segmentation. While returning accurate segmentations, these approaches require a tedious manual initialization at each pedicle and lack extensive validation. An alternative approach [18] developed a machine learning-based pedicle detection method to detect the lower end of left pedicles in L1, L2, and L3. However, they reported an accuracy of $48 \%$ for their best classifier.

In this study, a method for quasi-automated pedicle detection in frontal radiographs is described and evaluated in terms of sensitivity and precision compared to manual selection of the pedicles. The influence of manual annotations is also evaluated through a simulated reproducibility study. The detected pedicles were further evaluated via the obtained regressed measures of VAR, comparing to values obtained via manual pedicle selection and to the values extracted from 3D reconstruction of the spine.

\section{Materials and methods}

\section{Data}

A training dataset of 92 biplanar radiographs (EOS ${ }^{\mathrm{TM}}$ imaging system, Paris, France) of healthy and scoliotic patients (Cobb angles $3^{\circ}-93^{\circ}$, mean \pm SD: $42^{\circ} \pm 24^{\circ}$ ) and associated 3D spine reconstructions [3] was built retrospectively. At different steps of an iterative process, this dataset was used to statistically infer the approximate position of the pedicles.

A second dataset of 57 frontal radiographs-extracted from biplanar X-rays-of healthy and scoliotic subjects was built retrospectively for training and testing. Testing involved manual inputs to generate search areas for the pedicles, and automated post-processing to detect pedicles within the search areas and then to infer missing pedicles. Patients' characteristics are reported in Table 1. Patients suffering from very severe scoliosis, wearing braces or having metallic implants were not included in this dataset. For all radiographs, manual annotation of the pedicles was conducted by biomedical engineers specialized in spine imaging as well as 3D reconstruction of the spine using a previously validated method [3]. This study used previous data collection approved by the ethical committee, and patients or parents informed consent was obtained.

\section{Manual initialization}

First, a 2D model of the spine was estimated via manual drawing of the spinal curve and positioning of four vertebral endplates on the radiograph: the upper endplates of $\mathrm{T} 1$ and T4, and the lower endplates of T12 and L5 (Fig. 1b). From this initial model, probable pedicle positions were approximated by rectangular ROIs (Fig. 1c).

\section{Visual descriptors extraction}

After image enhancement using a combination of classical image filters, two types of visual descriptors were applied:
Table 1 Training and testing datasets characteristics.

(Ave $=$ average $)$

\begin{tabular}{|c|c|c|c|c|c|c|c|}
\hline & \multicolumn{3}{|c|}{ Number of cases } & \multicolumn{2}{|c|}{ Age (year) } & \multicolumn{2}{|c|}{ Cobb angle $\left(^{\circ}\right)$} \\
\hline & Female & Male & Total & Range & Ave & Range & Ave \\
\hline Group 1 (healthy) & 5 & 6 & 11 & $6-33$ & 18 & $0-8.8$ & 2.9 \\
\hline Group 2 (mild scoliosis) & 26 & 4 & 30 & $6-16$ & 11 & $10.1-19.7$ & 14.2 \\
\hline Group 3 (moderate scoliosis) & 13 & 3 & 16 & $6-16$ & 11 & $20.5-45.5$ & 27.9 \\
\hline
\end{tabular}



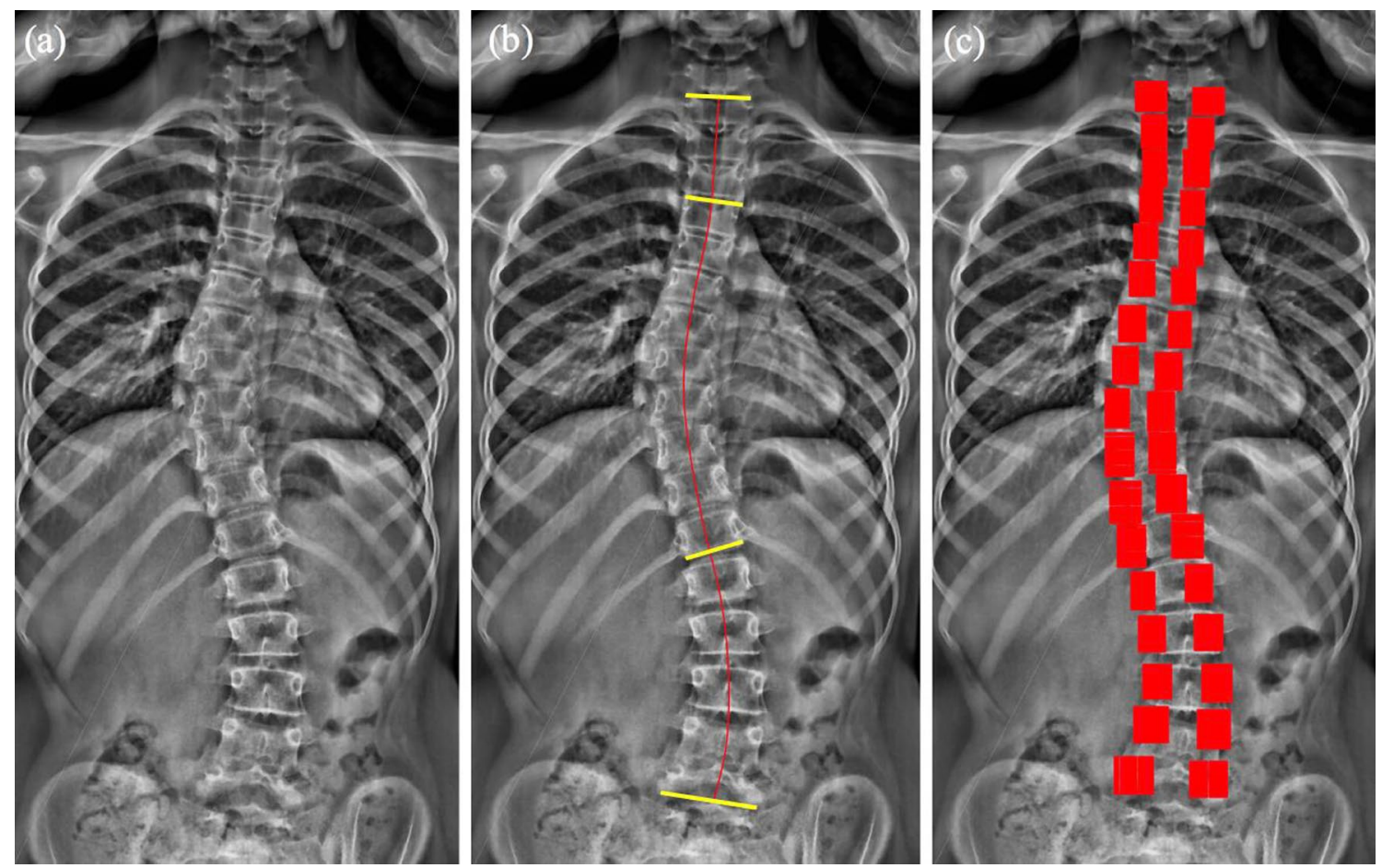

Fig. 1 2D statistical shape modeling. a Original frontal radiograph image. b Manually identified spinal line and four vertebral endplates: the upper endplates of $\mathrm{T} 1$ and $\mathrm{T} 4$ and lower endplates of $\mathrm{T} 12$

Histograms of oriented gradients (HOG) [19] and a specific configuration of contextual features (CF) [20] that combines local and remote visual information, around the pixel being analyzed.

\section{Pedicles detection}

Random Forest (RF) classifiers were trained for pedicle detection, using the HOG and CF features. RF is a supervised learning algorithm based on positive and negative instances, required in a training phase. RF has been chosen thanks to its ability to correctly classify instances based on a large amount of visual descriptors. The manually delineated pedicles in the training dataset were used as positive instances. Negative instances were randomly sampled from non-pedicle regions. Given the variability of pedicle shape and size, the spine was split into three regions (T1-T7, T8-T12, L1-L5). One RF classifier per region was learned. For training classifiers in T8-T12 and L1-L5 regions, the $\mathrm{X}$-rays were down-sampled by two, leading to approximately uniform pedicle sizes in pixels in all regions.

In the testing phase, within each ROI, a grid of $26 \times 24$ candidate pixels was automatically defined (Fig. 1c). As shown in Fig. 2a, b, the classifiers sometimes identified multiple clusters of pixels as belonging to one pedicle. In order to retain only one cluster, first mathematical morphology and L5. $\mathbf{c}$ Using the manual inputs in $\mathbf{b}$, a 2D model of the spine was obtained, from which approximate position of pedicles and search regions-of-interest (ROIs) are estimated (in red)

filtering was applied to group the detected pixels into at most two distinct clusters within each ROI (Fig. 2c). Then, cluster size and probability measures of its pixels, as assigned by the classifiers, helped retaining the most probable cluster (Fig. 2d).

The pedicle's center for each selected cluster in Fig. $2 d$ was calculated as the weighted mean of the cluster pixels coordinates, using probability measures as weights (Fig. 3a).

\section{Refinement stage}

An automatic refinement helped choosing the most robust pedicle centers and estimate the missing ones. First, false positives were discarded using a priori knowledge regarding inter-pedicular segment orientation and length. Then, the actual positions of these rejected false positives, as well as undetected pedicles, were inferred using the 2D spine shape model (Fig. 3b).

\section{Computation of vertebral orientations}

VARs were computed using the Stokes method [13], as described hereafter:

$$
\mathrm{VAR}=\arctan \left(\frac{w}{2 d} \times \frac{a-b}{a+b}\right)
$$



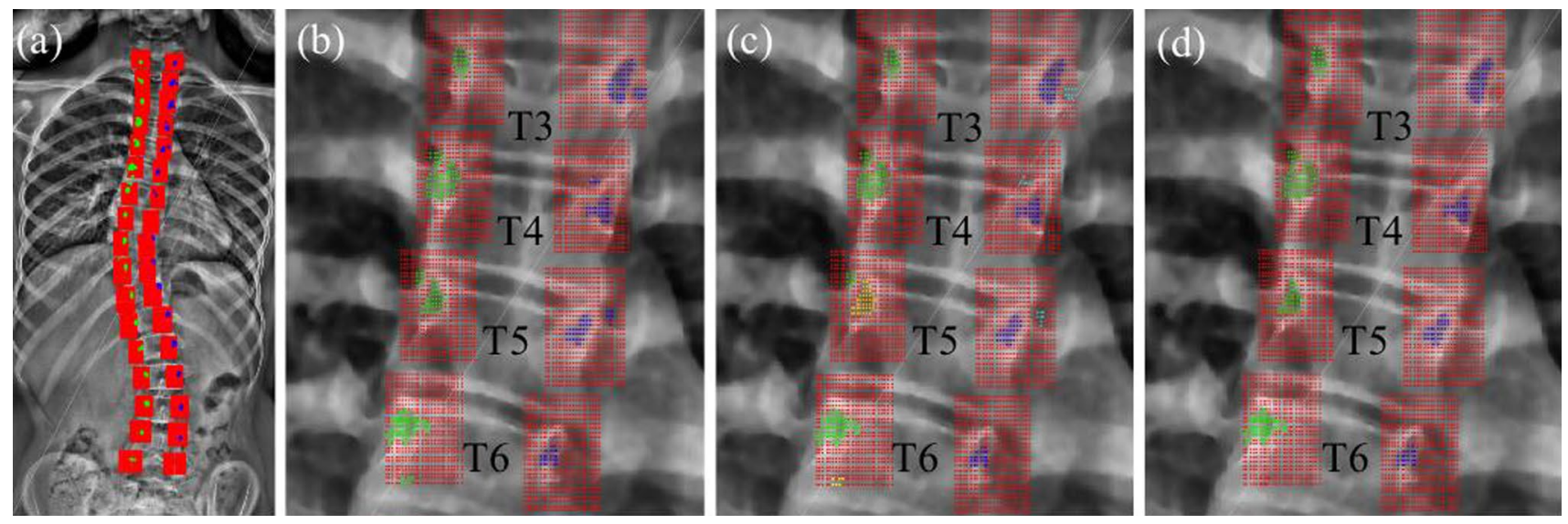

Fig. 2 Pedicle localization procedure. Candidate pixels are illustrated in red, and detected pedicle pixels are illustrated in blue (right) and green (left). a Red candidate pixels within bounding boxes are examined to classify them as belonging to a pedicle or not. b Output of the random forest classifiers, showing pixels probably belonging to a
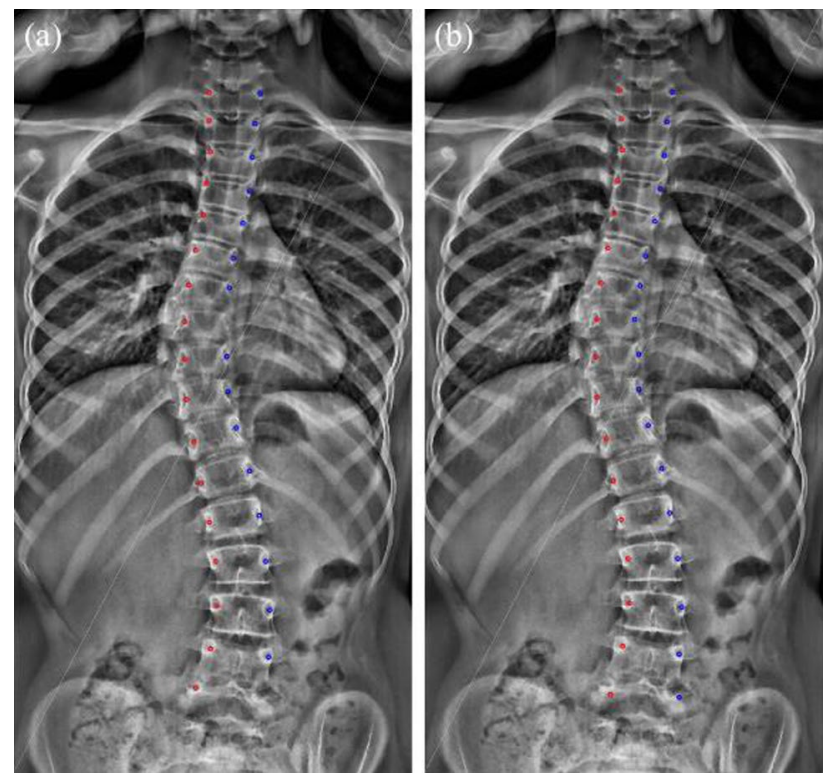

Fig. 3 Final pedicle centers detected by the proposed method. a Outcome of the pedicle center detection (a pedicle was not detected in T8 and L5) and $\mathbf{b}$ final results after the refinement stage used to infer missing pedicles and remove false positive detections (all pedicles were detected)

where $w$ and $2 d$ are the width and depth of the vertebra, respectively, while $a / b$ are the distances between left/right pedicles and vertebral body center. Values of the width-to-depth $(w / 2 d)$ ratio were derived from the patient dataset described in [3] (See Table 2). Manual VARs were calculated by considering a manually defined spinal curve passing through vertebral body centers. pedicle. $\mathbf{c}$ Using mathematical morphology filtering in each bounding box, detected pixels are grouped into a maximum of two clusters. $\mathbf{d}$ In each bounding box, the cluster with the highest score is kept and used to calculate pedicle centers

Table 2 Average values of vertebrae width-to-depth $(w / 2 d)$ ratios (in $\mathrm{mm}$ ) from $\mathrm{T} 1$ to $\mathrm{L} 5$

\begin{tabular}{ll}
\hline Vertebra & $\begin{array}{l}\text { Width-to- } \\
\text { depth ratio }\end{array}$ \\
\hline T1 & 1.24 \\
T2 & 1.08 \\
T3 & 0.97 \\
T4 & 0.89 \\
T5 & 0.83 \\
T6 & 0.78 \\
T7 & 0.75 \\
T8 & 0.73 \\
T9 & 0.71 \\
T10 & 0.70 \\
T11 & 0.69 \\
T12 & 0.69 \\
L1 & 0.69 \\
L2 & 0.70 \\
L3 & 0.71 \\
L4 & 0.73 \\
L5 & 0.79 \\
\hline
\end{tabular}

In addition, vertebral frontal rotations were also calculated by measuring the angle $\alpha$ between the inter-pedicular line $f$ and the image horizontal axis $h$ (Fig. 4).

\section{Analysis}

For all 57 subjects, positions of detected pedicle centers were compared to the positions of the manually delineated ones. A distance lower than $2 \mathrm{~mm}$ [21] was considered as a true positive (TP), otherwise as a false positive (FP). As $100 \%$ of pedicles were either detected or inferred, sensitivity was $100 \%$ 


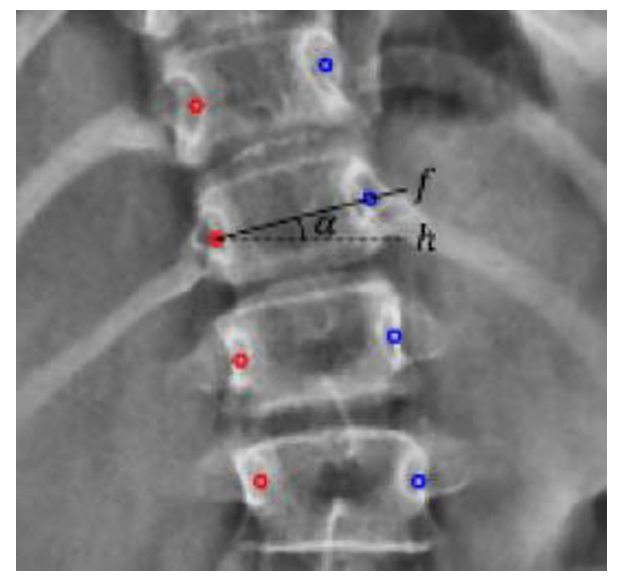

Fig. 4 Geometric basis for the measurement of vertebral frontal rotation $\alpha$

inherently. Precision was calculated as the rate of true positives $(\mathrm{TP} /(\mathrm{TP}+\mathrm{FP}) \times 100 \%)$. Quality of the detected pedicle center positions was evaluated in terms of root-mean-square error (RMSE) with respect to the manually delineated centers. Signed and unsigned frontal and axial rotation differences were computed, comparing detected and manually delineated pedicles. In addition, as 3D reconstructions were performed for all subjects, the calculated VARs were also compared to those obtained from the 3D models.

A subset of 20 scoliotic subjects $\left(\right.$ Cobb angle $=18.9^{\circ} \pm 7.5^{\circ}$ ) helped analyzing the influence of manual inputs on pedicle location. The manual inputs were modified ten times for each subject by adding a Gaussian noise with zero mean and standard deviation of $1 \mathrm{~mm}$ along the medio-lateral axis and $0.5 \mathrm{~mm}$ along the longitudinal axis. These ranges were extracted from a reproducibility analysis performed for this study based on six subjects and two operators who replicated twice their manual inputs. The proposed sensitivity study enabled us to investigate a larger number of subjects and repetitions $(N=200)$. Standard deviations of inferred pedicle locations along both axes were quantified for each subject and averaged on the full subset.

\section{Results}

Pedicle detection precisions were $83 \%, 85 \%$, and $86 \%$, respectively, for T1-T7, T8-T12 and lumbar regions. Detailed information is presented in Table 3 .
Table 4 Pedicle position differences between automatic detection and manual delineation (RMSE in $\mathrm{mm}$ )

\begin{tabular}{llll}
\hline Vertebral level & Mean & SD & Median \\
\hline T1 & 1.7 & 1.8 & 0.7 \\
T2 & 1.1 & 1.0 & 0.7 \\
T3 & 1.1 & 1.1 & 0.7 \\
T4 & 1.0 & 1.2 & 0.6 \\
T5 & 1.0 & 1.1 & 0.6 \\
T6 & 1.2 & 1.2 & 0.8 \\
T7 & 1.1 & 1.4 & 0.6 \\
T8 & 1.0 & 1.1 & 0.6 \\
T9 & 1.2 & 1.3 & 0.7 \\
T10 & 1.3 & 1.4 & 0.8 \\
T11 & 1.3 & 1.2 & 0.9 \\
T12 & 1.1 & 1.0 & 0.9 \\
L1 & 0.9 & 0.7 & 0.7 \\
L2 & 0.8 & 0.7 & 0.6 \\
L3 & 0.8 & 0.6 & 0.6 \\
L4 & 1.0 & 1.1 & 0.7 \\
L5 & 2.2 & 1.9 & 1.7 \\
Ave. (T1-L5) & 1.2 & 1.2 & 0.7 \\
\hline
\end{tabular}

Table 4 reports the RMSE distances between detected pedicles and manually delineated ones. A mean RMSE \pm SD of $1.2 \pm 1.2 \mathrm{~mm}$ was achieved over all vertebrae.

Table 5 reports the vertebral frontal rotation difference values. The average signed error $\pm \mathrm{SD}$ was $-0.2^{\circ} \pm 3.5^{\circ}$, and the average absolute frontal rotation difference was $2.4^{\circ} \pm 2.6^{\circ}$.

Table 6 reports the VAR difference values when using the Stokes method for pedicles from the proposed algorithm and the manual delineations. The average signed error \pm SD was $-0.2^{\circ} \pm 3.4^{\circ}$.

Table 7 reports the VAR difference values between the proposed method using the Stokes method and the 3D spine models. The average of signed differences was $0.0^{\circ} \pm 4.4^{\circ}$.

Figure 5 presents the Bland-Altman plots for the average VAR difference values between the proposed algorithm and the 3D spine reconstructions for the apical, and two upper/ lower junctional vertebrae of 46 scoliotic patients with the lowest SD of $2.5^{\circ}$ for apical vertebrae.

For the same dataset, Table 8 reports the average VAR differences within and outside the main scoliotic curve. The

Table 3 The algorithm performance metric on the detected pedicles within the confidence interval ( $2 \mathrm{~mm}$ to the manual reference)

\begin{tabular}{|c|c|c|c|c|c|c|c|c|c|c|c|c|c|c|c|c|c|c|}
\hline Vertebral level & $\mathrm{T} 1$ & $\mathrm{~T} 2$ & $\mathrm{~T} 3$ & $\mathrm{~T} 4$ & T5 & T6 & $\mathrm{T} 7$ & $\mathrm{~T} 8$ & T9 & $\mathrm{T} 10$ & $\mathrm{~T} 11$ & $\mathrm{~T} 12$ & $\mathrm{~L} 1$ & L2 & L3 & $\mathrm{L} 4$ & L5 & Ave. (T1-L5) \\
\hline Precision (\%) & 68 & 85 & 85 & 90 & 84 & 83 & 85 & 89 & 84 & 79 & 84 & 88 & 92 & 95 & 95 & 89 & 57 & 84 \\
\hline
\end{tabular}


Table 5 Differences of vertebral frontal rotation values (proposed detection method vs. manual 2D delineation)

\begin{tabular}{|c|c|c|c|c|c|c|c|c|c|c|c|c|c|c|c|c|c|c|}
\hline Vertebrae & $\mathrm{T} 1$ & $\mathrm{~T} 2$ & T3 & $\mathrm{T} 4$ & $\mathrm{~T} 5$ & T6 & $\mathrm{T} 7$ & $\mathrm{~T} 8$ & T9 & $\mathrm{T} 10$ & $\mathrm{~T} 11$ & $\mathrm{~T} 12$ & L1 & $\mathrm{L} 2$ & L3 & L4 & L5 & $\overline{\text { Average }}$ \\
\hline \multicolumn{19}{|c|}{ Unsigned differences $\left({ }^{\circ}\right)$} \\
\hline Mean & 2.2 & 1.6 & 2.1 & 2.1 & 3.1 & 2.7 & 2.4 & 2.2 & 3.4 & 3.5 & 2.5 & 2.6 & 2.0 & 1.6 & 1.6 & 1.7 & 2.7 & 2.4 \\
\hline SD & 2.4 & 1.6 & 2.4 & 2.3 & 2.9 & 2.5 & 2.5 & 2.4 & 3.9 & 3.2 & 3.0 & 2.6 & 1.8 & 1.9 & 1.5 & 2.4 & 2.8 & 2.6 \\
\hline Median & 1.2 & 1.0 & 1.3 & 1.4 & 2.1 & 2.1 & 1.9 & 1.5 & 2.0 & 2.8 & 1.5 & 2.0 & 1.4 & 1.2 & 1.3 & 0.9 & 1.4 & 1.5 \\
\hline Vertebrae & $\mathrm{T} 1$ & $\mathrm{~T} 2$ & $\mathrm{~T} 3$ & $\mathrm{~T} 4$ & $\mathrm{~T} 5$ & T6 & $\mathrm{T} 7$ & $\mathrm{~T} 8$ & $\mathrm{~T} 9$ & $\mathrm{~T} 10$ & $\mathrm{~T} 11$ & $\mathrm{~T} 12$ & $\mathrm{~L} 1$ & L2 & L3 & $\mathrm{L} 4$ & L5 & Total \\
\hline \multicolumn{19}{|c|}{ Signed differences $\left({ }^{\circ}\right)$} \\
\hline Mean & -1.5 & -0.1 & -0.1 & 0.2 & 0.0 & 0.1 & 0.0 & 0.1 & -2.6 & -0.9 & -0.6 & -0.1 & 0.2 & -0.2 & 0.1 & 0.4 & 0.7 & -0.2 \\
\hline SD & 2.9 & 2.3 & 3.2 & 3.1 & 4.3 & 3.7 & 3.5 & 3.3 & 4.5 & 4.7 & 3.9 & 3.7 & 2.7 & 2.5 & 2.2 & 2.9 & 3.8 & 3.5 \\
\hline Median & -0.4 & 0.2 & 0.1 & 0.2 & -0.6 & 0.0 & 0.6 & 0.4 & -0.9 & -0.5 & 0.0 & 0.2 & 0.7 & 0.1 & 0.0 & 0.3 & -0.3 & 0.0 \\
\hline
\end{tabular}

Table 6 Differences of vertebral axial rotation values (VAR), using the Stokes method (proposed detection method vs. manual 2D delineation)

\begin{tabular}{|c|c|c|c|c|c|c|c|c|c|c|c|c|c|c|c|c|c|c|}
\hline Vertebrae & $\mathrm{T} 1$ & $\mathrm{~T} 2$ & $\mathrm{~T} 3$ & $\mathrm{~T} 4$ & T5 & T6 & $\mathrm{T} 7$ & $\mathrm{~T} 8$ & T9 & $\mathrm{T} 10$ & $\mathrm{~T} 11$ & $\mathrm{~T} 12$ & L1 & L2 & L3 & L4 & L5 & Ave \\
\hline \multicolumn{19}{|c|}{ Unsigned differences $\left({ }^{\circ}\right)$} \\
\hline Mean & 3.3 & 2.7 & 2.5 & 2.5 & 2.5 & 2.4 & 2.2 & 2.2 & 1.9 & 2.3 & 2.0 & 1.4 & 1.2 & 0.9 & 0.9 & 1.1 & 2.5 & 2.0 \\
\hline SD & 3.9 & 3.0 & 3.1 & 4.2 & 2.9 & 2.3 & 4.2 & 2.5 & 2.5 & 3.3 & 2.9 & 2.0 & 1.1 & 0.8 & 0.7 & 1.0 & 2.2 & 2.8 \\
\hline Median & 1.7 & 2.1 & 1.4 & 1.2 & 1.7 & 2.0 & 0.9 & 1.4 & 1.1 & 1.1 & 1.0 & 0.7 & 1.0 & 0.8 & 0.8 & 0.8 & 1.8 & 1.1 \\
\hline \multicolumn{19}{|c|}{ Signed differences $\left({ }^{\circ}\right)$} \\
\hline Mean & 1.6 & -1.0 & -0.6 & -1.2 & 0.0 & -0.3 & -1.3 & 0.4 & 0.2 & -1.1 & -1.1 & -0.4 & -0.1 & 0.1 & 0.3 & 0.1 & 1.1 & -0.2 \\
\hline SD & 4.9 & 3.9 & 3.9 & 4.7 & 3.9 & 3.4 & 4.5 & 3.3 & 3.1 & 3.9 & 3.3 & 2.4 & 1.6 & 1.2 & 1.1 & 1.5 & 3.2 & 3.4 \\
\hline Median & 0.5 & -0.3 & -0.4 & -0.5 & 0.2 & -0.4 & -0.3 & 1.1 & 0.1 & 0.0 & 0.2 & -0.1 & 0.3 & 0.1 & 0.5 & 0.2 & 1.0 & 0.1 \\
\hline
\end{tabular}

Table 7 Differences of vertebral axial rotation values (VAR), using the Stokes method with proposed detection method versus 3D reconstruction

\begin{tabular}{|c|c|c|c|c|c|c|c|c|c|c|c|c|c|c|c|c|c|c|}
\hline Vertebrae & $\mathrm{T} 1$ & $\mathrm{~T} 2$ & $\mathrm{~T} 3$ & $\mathrm{~T} 4$ & $\mathrm{~T} 5$ & T6 & $\mathrm{T} 7$ & $\mathrm{~T} 8$ & T9 & $\mathrm{T} 10$ & $\mathrm{~T} 11$ & $\mathrm{~T} 12$ & L1 & L2 & L3 & L4 & L5 & Ave \\
\hline \multicolumn{19}{|c|}{ Unsigned differences $\left({ }^{\circ}\right)$} \\
\hline Mean & 5.0 & 3.4 & 3.9 & 3.9 & 3.6 & 3.1 & 3.0 & 3.0 & 2.9 & 3.3 & 2.6 & 2.2 & 1.8 & 1.7 & 1.8 & 1.9 & 3.4 & 3.0 \\
\hline SD & 4.8 & 3.6 & 3.8 & 4.8 & 3.4 & 3.0 & 3.8 & 3.2 & 3.1 & 3.4 & 2.8 & 2.1 & 1.4 & 2.2 & 2.1 & 1.7 & 2.7 & 3.3 \\
\hline Median & 3.0 & 2.1 & 2.5 & 2.6 & 2.9 & 2.3 & 1.7 & 1.7 & 2.4 & 2.1 & 1.6 & 1.2 & 1.5 & 1.2 & 1.3 & 1.6 & 3.1 & 1.9 \\
\hline \multicolumn{19}{|c|}{ Signed differences $\left({ }^{\circ}\right)$} \\
\hline Mean & 1.7 & -1.8 & -1.7 & -2.5 & -0.7 & -0.2 & -0.9 & 1.6 & 1.2 & -0.3 & -0.4 & -0.9 & 0.5 & 0.9 & 1.1 & 0.3 & 1.9 & 0.0 \\
\hline SD & 6.8 & 4.7 & 5.2 & 5.6 & 4.9 & 4.3 & 4.7 & 4.1 & 4.0 & 4.8 & 3.8 & 2.9 & 2.2 & 2.6 & 2.5 & 2.5 & 3.9 & 4.4 \\
\hline Median & 1.0 & -1.5 & -1.8 & -2.0 & -0.8 & -0.4 & 0.3 & 1.2 & 1.6 & 0.7 & 0.2 & -0.8 & 0.6 & 0.8 & 0.9 & 0.2 & 1.9 & 0.2 \\
\hline
\end{tabular}

average of signed differences for these first regions were $0.5^{\circ} \pm 3.8^{\circ}$ and $-0.2^{\circ} \pm 4.4^{\circ}$, respectively.

The sensitivity analysis led to a global uncertainty on pedicle location of $2 \mathrm{~mm}$ (twice the average standard deviation observed on the full subset) on the longitudinal axis and $1.6 \mathrm{~mm}$ on the medio-lateral axis. Looking at each subject independently, an uncertainty range from 0.9 to $2.8 \mathrm{~mm}$ on the frontal axis and from 1.2 to $3.4 \mathrm{~mm}$ for the longitudinal axis were obtained.

\section{Discussion}

Vertebral axial rotation is of primary importance for analyzing a scoliotic spine. Although ideally VAR can be obtained in standing position from biplanar radiographs and associated 3D reconstructions, single frontal radiographs are still widely used in clinics, with a need for time-consuming manual localization of all pedicles. In this work, a quasi-automated pedicle localization method was proposed, based on an original combination of machine 
Fig. 5 Bland-Altman plot for the axial rotation of apical vertebrae (a), lower junctional vertebrae (b), and upper junctional vertebrae (c) of 46 scoliotic patients as illustrated in black circles. ( $\mathrm{Alg}=$ algorithm, $3 \mathrm{D}=3 \mathrm{D}$ reconstruction)
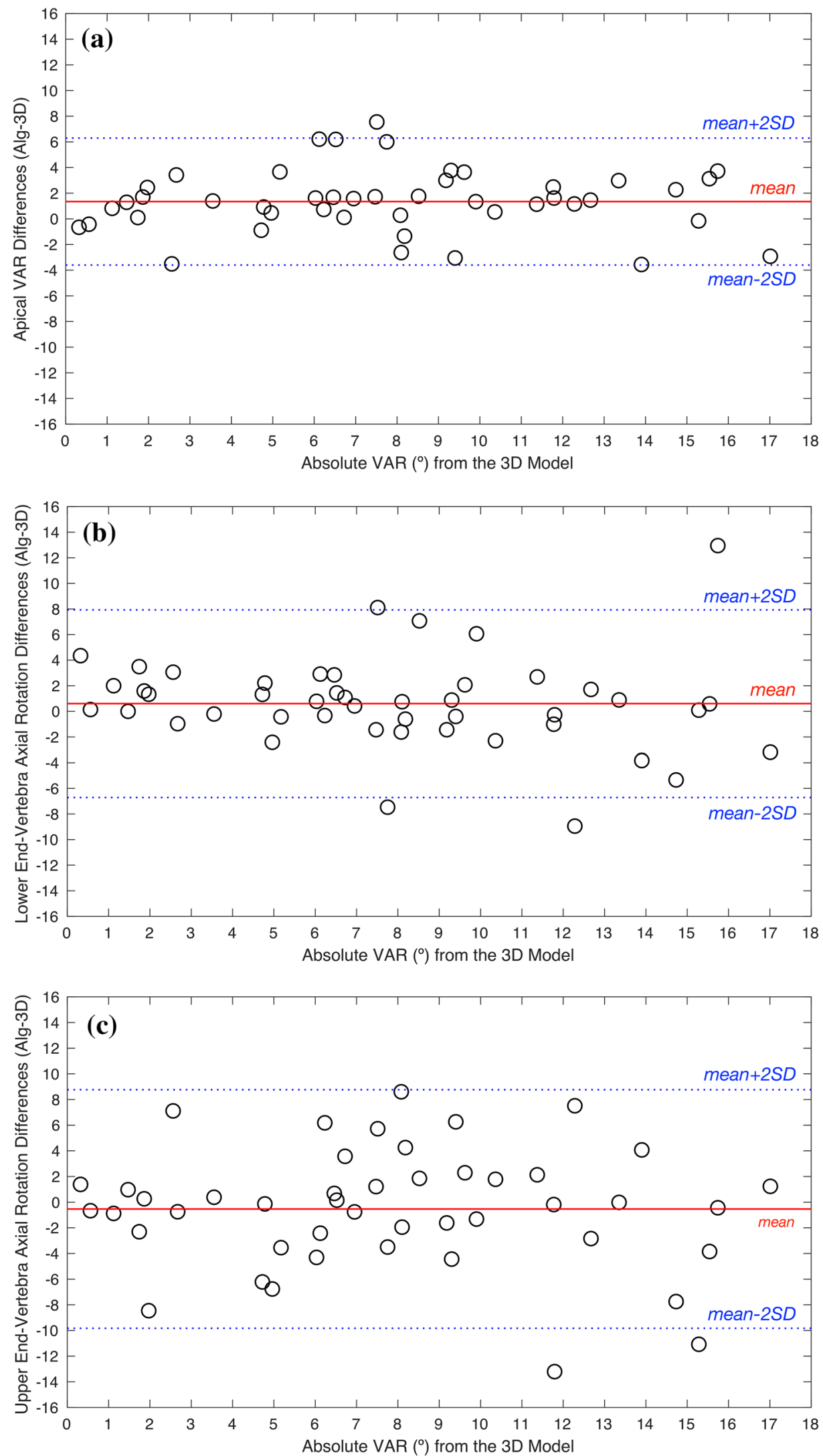
Table 8 Difference of VAR values (proposed algorithm-3D reconstruction) in $\left(^{\circ}\right)$

\begin{tabular}{llc}
\hline Vertebrae & $\begin{array}{l}\text { Within the main scoliotic } \\
\text { curve }\end{array}$ & $\begin{array}{l}\text { Outside } \\
\text { the main } \\
\text { scoliotic } \\
\text { curve }\end{array}$ \\
\hline Mean & 0.5 & -0.2 \\
SD & 3.8 & 4.4 \\
Median & 0.7 & -0.1 \\
\hline
\end{tabular}

learning, image processing and statistical shape modeling. Stokes method was then used for VAR estimation.

The original formulation of visual descriptors used by the classifiers proved to be very efficient for pedicle detection. On the other hand, the refinement strategy based on statistical shape modeling and image processing made the algorithm robust to false detections. Exploiting a 2D statistical shape model also provided the a priori knowledge required for estimating missing pedicles; hence, a $100 \%$ pedicle center detection sensitivity was achieved. When considering a distance threshold of $2 \mathrm{~mm}$, a localization precision of $84 \%$ was achieved after the refinement stage, which is promising compared to the respective sensitivity and precision values of $77 \%$ and $88 \%$ before the refinement stage. Precision decreased after refinement because statistical estimates of missing values were then taken into account. Overall, after the refinement step, average pedicle localization RMSE was limited to $1.2 \pm 1.2 \mathrm{~mm}$. As this method has been trained on frontal radiographs acquired with the EOS system, a replication study with maybe another training might be necessary before applying it on radiographs acquired with a different radiographic system.

To the best of our knowledge, the presented method has been more extensively validated than the methods available in the literature, by considering each thoracic and lumbar vertebra on a reasonably sized cohort including pathologic subjects. For instance, Doré et al. [14] tested their approach of pedicle segmentation and vertebral frontal rotation assessment on T1-L4 of only five scoliotic patients. In [16], VARs were estimated from segmented pedicles (T4-L4) on frontal radiographs of only 12 scoliotic subjects, and in [18], the authors focused on L1-L3 left pedicles of 21 subjects.

In addition, these methods were based on either an extensive manual initialization of each pedicle location $[14,16]$ or a pixel-wise examination of the image [18] (resulting in a potentially large computational cost). In [14], poorly visible pedicles were a priori discarded, and in [16] all pedicles in their dataset were visible. The proposed method did not have these limitations, as a fast initialization was proposed with few landmarks for the full spine, and the derived statistical shape model could deal with poorly visible pedicles. In addition, the sensitivity of pedicle location to the limited manual inputs has been analyzed. It appeared to be very low, with a global uncertainty of pedicle location of less than $2 \mathrm{~mm}$ in both longitudinal and medio-lateral axes.

The proposed method showed limited number of false positives in the lumbar region. The thoracic region was more challenging due to anatomical structure superimpositions, which could generate shadow artifacts with visual appearance similar to pedicles or cause poor contrast. Axial rotation could also be higher in the thoracic than in the lumbar region, significantly altering pedicle projection in frontal radiographs. Based on our experiments, missed-detections could typically occur on poorly visible pedicles or from misplacement of the search area during the initialization.

Moreover, when using Stokes method for VAR estimation, comparison between quasi-automated pedicle detection and manual pedicle delineation showed a mean difference of $1.2 \pm 1.2 \mathrm{~mm}$, for all vertebrae. These differences were within reported uncertainty of Stokes method. Therefore, from a single frontal X-Ray, VAR could be estimated with the proposed method.

Finally, among the works aiming to infer VAR from frontal images, none has compared their results with VAR obtained from a 3D reconstruction of the spine. However, this is essential to assess the relevance of such measurement from a single frontal X-Ray. The bias of signed values was negligible (Table 7). The standard deviation of VAR differences between the proposed algorithm and the $3 \mathrm{D}$ reconstruction was $1^{\circ}$ higher than the standard deviation obtained when comparing our method to the manual $2 \mathrm{D}$ method $\left(4.4^{\circ}\right.$ and $3.4^{\circ}$, respectively). One reason is that in the Stokes method, the vertebral width-to-depth ratio was set to average values, and hence not patient specific. Therefore, VARs computed from the proposed method were affected by vertebral shape variability, which is accounted for in 3D reconstructions. Regarding VAR differences between the proposed method and 3D reconstructions of specific vertebrae in the main curve (apical vertebra and junctional vertebrae), the average signed error of $1.3^{\circ} \pm 2.5^{\circ}$ was achieved for apical vertebrae. This value for the upper and lower end vertebrae was $-0.5^{\circ} \pm 4.6^{\circ}$ and $0.6^{\circ} \pm 3.7^{\circ}$, respectively. The error was higher in T1-T7, and hence higher average error for upper end vertebrae was expected.

\section{Conclusion}

The proposed quasi-automatic pedicle detection method enables a reliable vertebral rotations estimation from a single frontal radiograph when $3 \mathrm{D}$ reconstruction is not available. The proposed method can be used as a standalone vertebral rotation estimation tool, and has potential applications toward the automation of 3D spine reconstructions. 
The proposed method could also be extended to other anatomical structures in the spine.

Acknowledgements The authors are grateful to the ParisTech BiomecAM chair program on subject-specific musculoskeletal modeling for funding (with the support of ParisTech and Yves Cotrel Foundations, Société Générale, Proteor and Covea).

\section{Compliance with ethical standards}

Conflict of interest The authors have no conflicts of interest to declare.

\section{References}

1. Illés TS, Burkus M, Somoskeőy S et al (2017) The horizontal plane appearances of scoliosis: what information can be obtained from top-view images? Int Orthop. https://doi.org/10.1007/s0026 4-017-3548-5

2. Illés TS, Lavaste F, Dubousset JF (2019) The third dimension of scoliosis: the forgotten axial plane. Orthop Traumatol Surg Res 105:351-359. https://doi.org/10.1016/j.otsr.2018.10.021

3. Humbert L, De Guise JA, Aubert B et al (2009) 3D reconstruction of the spine from biplanar $\mathrm{X}$-rays using parametric models based on transversal and longitudinal inferences. Med Eng Phys 31:681-687. https://doi.org/10.1016/j.medengphy.2009.01.003

4. Vergari C, Gajny L, Courtois I et al (2019) Quasi-automatic early detection of progressive idiopathic scoliosis from biplanar radiography: a preliminary validation. Eur Spine J. https://doi. org/10.1007/s00586-019-05998-z

5. Skalli W, Vergari C, Ebermeyer E et al (2017) Early detection of progressive adolescent idiopathic scoliosis. Spine 42:823-830. https://doi.org/10.1097/BRS.0000000000001961

6. Mainard-Simard L, Lan L, Fort D (2017) The advantages of 3D imagery in diagnosing and supervising children's and teenagers' scoliosis. Arch Pédiatr 24:1029-1035 (in French). https://doi. org/10.1016/j.arcped.2017.07.008

7. Ferrero E, Lafage R, Diebo BG et al (2017) Tridimensional analysis of rotatory subluxation and sagittal spinopelvic alignment in the setting of adult spinal deformity. Spine Deform 5:255-264. https://doi.org/10.1016/j.jspd.2017.01.003

8. Rehm J, Germann T, Akbar M et al (2017) 3D-modeling of the spine using EOS imaging system: inter-reader reproducibility and reliability. PLoS One 12:e171258. https://doi.org/10.1371/journ al.pone. 0171258

9. Newton PO, Khandwala Y, Bartley CE et al (2016) New EOS imaging protocol allows a substantial reduction in radiation exposure for scoliosis patients. Spine Deform 4:138-144. https://doi. org/10.1016/j.jspd.2015.09.002

10. Lebel DE, Al-Aubaidi Z, Shin E-J et al (2013) Three dimensional analysis of brace biomechanical efficacy for patients with
AIS. Eur Spine J 22:2445-2448. https://doi.org/10.1007/s0058 6-013-2921-3

11. Courvoisier A, Garin C, Vialle R, Kohler R (2015) The change on vertebral axial rotation after posterior instrumentation of idiopathic scoliosis. Child Nerv Syst 31:2325-2331. https://doi. org/10.1007/s00381-015-2891-3

12. Ilharreborde B, Steffen JS, Nectoux E et al (2011) Angle measurement reproducibility using EOS three-dimensional reconstructions in adolescent idiopathic scoliosis treated by posterior instrumentation. Spine 36:E1306-E1313. https://doi.org/10.1097/BRS.0b013 e3182293548

13. Stokes IA, Bigalow LC, Moreland MS (1986) Measurement of axial rotation of vertebrae in scoliosis. Spine 11:213-218

14. Doré V, Duong L, Cheriet F, Cheriet M (2007) Towards segmentation of pedicles on posteroanterior $\mathrm{X}$-ray views of scoliotic patients. In: Kamel M, Campilho A (eds) Image analysis and recognition: 4th international conference, ICIAR 2007, Montreal, proceedings. Springer, Berlin, pp 1028-1039

15. Zhang J, Shi X, Wang Y, et al (2010) Snake-based approach for segmenting pedicles in radiographs and its application in threedimensional vertebrae reconstruction. In: 2010 IEEE international conference on image processing. IEEE, pp 2569-2572

16. Zhang J, Lou E, Hill DL et al (2010) Computer-aided assessment of scoliosis on posteroanterior radiographs. Med Biol Eng Comput 48:185-195. https://doi.org/10.1007/s11517-009-0556-7

17. Kumar S, Nayak KP, Hareesh KS (2012) Semiautomatic method for segmenting pedicles in vertebral radiographs. Proc Technol 6:39-48. https://doi.org/10.1016/j.protcy.2012.10.006

18. Cunha P, Moura DC, Barbosa JG (2012) Pedicle detection in planar radiographs based on image descriptors. In: Campilho A, Kamel M (eds) Image analysis and recognition: 9th international conference, ICIAR 2012, Aveiro, proceedings, part II. Springer, Berlin, pp 278-285

19. Dalal N, Triggs B (2005) Histograms of oriented gradients for human detection. In: Proceedings-2005 IEEE computer society conference on computer vision and pattern recognition. CVPR, Washington, DC, pp 886-893

20. Ebrahimi S, Gajny L, Skalli W, Angelini E (2019) Vertebral corners detection on sagittal $\mathrm{x}$-rays based on shape modelling, random forest classifiers and dedicated visual features. Comput Methods Biomech Biomed Eng Imaging Vis 7:132-144. https:// doi.org/10.1080/21681163.2018.1463174

21. André B, Dansereau J, Labelle H (1992) Effect of radiographic landmark identification errors on the accuracy of three-dimensional reconstruction of the human spine. Med Biol Eng Comput 30:569-575. https://doi.org/10.1007/BF02446787

\section{Affiliations}

\section{Shahin Ebrahimi ${ }^{1} \cdot$ Laurent Gajny $^{1}{ }^{10} \cdot$ Claudio Vergari $^{1} \cdot$ Elsa D. Angelini ${ }^{2,3} \cdot$ Wafa Skalli $^{1}$}

1 Institut de Biomécanique Humaine Georges Charpak, Arts et Métiers Institute of Technology, Paris, France

2 LTCI, Department Image-Data-Signal, Telecom ParisTech, Paris, France
3 ITMAT Data Science Group, NIHR Imperial BRC, Imperial College London, London, UK 